\title{
STATISTICAL STUDY OF THE RECONNECTION RATE IN SOLAR FLARES OBSERVED WITH YOHKOH/SXT
}

\author{
Kaori Nagashima ${ }^{1}$ and Takaaki Yokoyama ${ }^{2}$
}

\begin{abstract}
We report a statistical study of flares observed with the Soft X-ray Telescope (SXT) onboard Yohkoh in the year of 2000. We measure physical parameters of 77 flares, such as the temporal scale, the size, and the magnetic flux density and find that the sizes of flares tend to be distributed more broadly as the GOES class becomes weaker and that there is a lower limit of magnetic flux density that depends on the GOES class. We also examine the relationship between these parameters and find weak correlation between temporal and spatial scales of flares. We estimate reconnection inflow velocity, coronal Alfvén velocity, and reconnection rate using above observed values. The inflow velocities are distributed from a few $\mathrm{km} \mathrm{s}^{-1}$ to several tens $\mathrm{km} \mathrm{s}^{-1}$ and the Alfvén velocities in the corona are in the range from $10^{3}$ to $10^{4} \mathrm{~km} \mathrm{~s}^{-1}$. Hence the reconnection rate is $10^{-3}-10^{-2}$. We find that the reconnection rate in a flare tends to decrease as the GOES class of the flare increases. This value is within one order of magnitude from the theoretical maximum value predicted by the Petschek model, although the dependence of the reconnection rate on the magnetic Reynolds number tends to be stronger than that in the Petschek model.
\end{abstract}

Subject headings: Sun: corona - Sun: flares — Sun: magnetic fields

\section{INTRODUCTION}

In the energy release process in solar flares, magnetic reconnection is generally considered to play a key role. The reconnection rate is an important quantity, because it puts critical restrictions on the reconnection model. It is defined as $M_{\mathrm{A}} \equiv V_{\mathrm{in}} / V_{\mathrm{A}}$ in non-dimensional

\footnotetext{
${ }^{1}$ Department of Astronomy, Kyoto University, Sakyo-ku, Kyoto 606-8502, Japan; kaorin@kwasan.kyotou.ac.jp

${ }^{2}$ Department of Earth and Planetary Science, University of Tokyo, Hongo, Bunkyo-ku, Tokyo 113-0033, Japan
} 
form, where $V_{\text {in }}$ is the velocity of the reconnection inflow and $V_{\mathrm{A}}$ is the Alfvén velocity. It gives the normalized value of the reconnected flux per unit time. In spite of its importance, what determines the reconnection rate in flares is still a question. In the steady reconnection model of Sweet (1958) and Parker (1957), the reconnection rate is $M_{\mathrm{A}}=\left(R_{\mathrm{m}}\right)^{-1 / 2}$, where $R_{\mathrm{m}}=\left(V_{\mathrm{A}} L / \eta\right)$ is the magnetic Reynolds number defined with the Alfvén velocity and $\eta$ is the magnetic diffusivity. In the solar corona, if the resistivity is attributed to the Coulomb collision (Spitzer 1956), the typical value of $R_{\mathrm{m}}$ is $R_{\mathrm{m}} \sim 10^{14}$, which means $M_{\mathrm{A}} \sim 10^{-7}$ and estimated timescale of flare is about one year. It is, of course, too slow to explain flares whose timescale is about $10^{2}-10^{3}$ seconds. On the other hand, Petschek (1964) pointed out that the previous model lacks the effect of wave and suggested his model with $M_{\mathrm{A}} \lesssim \pi /\left(8 \ln \left(8 R_{\mathrm{m}}\right)\right)$ . The special feature of this model is that $M_{\mathrm{A}}$ has weak dependence on $R_{\mathrm{m}}$. In this model $M_{\mathrm{A}} \lesssim 10^{-2}$ when $R_{\mathrm{m}} \sim 10^{14}$ and the estimated timescale is consistent with observed value (see also Nitta 2004).

The Sweet-Parker model and the Petschek model shown above are steady reconnection models. The physics of nonsteady reconnection is not established clearly. Shibata \& Tanuma (2001) discussed the plasmoid-induced-reconnection model. Since flares are nonsteady phenomena, they suggested possibility that the reconnection rate in a flare is determined by the nonsteady and dynamic processes, such as plasmoid ejections. Another uncertainty in the model of the reconnection mechanism is uncertainty of the magnetic Reynolds number $R_{\mathrm{m}}$. It was suggested that finite levels of MHD turbulence can lead to increase of the magnetic diffusivity, i.e., decrease of the effective magnetic Reynolds number (Matthaeus \& Lamkin 1985; Tajima \& Shibata 1997, §3.3).

Though there are some observational case studies about the reconnection rate, statistical studies are very few so far. Dere (1996) examined the spatial and temporal scales of reconnection events in the solar atmosphere described in the literature and found that the reconnection rate is on the order of 0.001 - 0.1. Tsuneta (1996) analyzed the temperature structure of 1992 February 21 LDE flare in detail and found that the reconnection rate is 0.07. He assumed isothermal switch-off shock and used the condition of the shock to derive the reconnection rate. The flare on 1992 January 13 was analyzed by Tsuneta et al. (1997) and the reconnection rate in this flare was estimated at 0.06. Ohyama \& Shibata (1997) and Ohyama \& Shibata (1998) studied 1993 November 11 flare and 1992 October 5 flare, respectively. They found the value of $0.003-0.2$ in preflare and impulsive phase, and 0.0002 - 0.0003 in gradual phase. Isobe et al. (2002b) analyzed the decay phase of an LDE flare on 1997 May 12 and estimated the reconnection rate at 0.001-0.01 using the energy release rate and the reconnected magnetic flux. Using the same method as the previous one, Isobe et al. (2002a) analyzed 7 flares and 2 giant arcades and found that the reconnection rate is 0.001 - 0.1. Moreover, analyzing the impulsive phase of three two-ribbon flares, Isobe et al. (2005) 
obtained the value of $0.015-0.071$.

Recently, direct measurements of the reconnection inflow were reported. Yokoyama et al. (2001) found an evidence of reconnection inflow and derived the reconnection rate as $0.001-0.03$. By the same method, Narukage \& Shibata (2006) reported 6 inflow events and found the reconnection rate is $0.001-0.07$. Lin et al. (2005) studied an eruptive limb event occurred on 2003 November 18 and reported the reconnection rate is in the range from 0.01 to 0.23 . Note also that they found the reconnection inflow using the Doppler shift.

In this paper, we report a statistical study of flares that occurred in the year of 2000 observed with Yohkoh/SXT. We determine several physical quantities of each flare and try to estimate the reconnection rate in solar flares. Our aim is to find statistical tendency of the physical parameters. In $\S 2$, we describe the data and the method of analysis. In $\S 3$, we show results. Discussion and conclusion are given in $\S 4$ and $\S 5$, respectively.

\section{DATA ANALYSIS}

\subsection{Inflow Velocity}

The amount of energy released during a flare $E_{\text {flare }}$ can be explained by the magnetic energy stored in the solar atmosphere;

$$
E_{\text {flare }} \sim E_{\text {mag }}=\frac{B_{\text {cor }}^{2}}{8 \pi} L^{3},
$$

where $L$ is the characteristic size of the flare and $B_{\text {cor }}$ is the characteristic magnetic flux density in the corona. Since the released magnetic energy balances the energy flowing into the reconnection region, we can describe the energy release rate as

$$
\left|\frac{d E_{\mathrm{mag}}}{d t}\right| \simeq 2 \times \frac{B_{\mathrm{cor}}^{2}}{4 \pi} V_{\mathrm{in}} \times L^{2},
$$

where $V_{\text {in }}$ is the inflow velocity of the plasma. Therefore, the time required for the energy inflow to supply the flare energy is estimated as

$$
\tau_{\text {flare }} \sim E_{\text {flare }}\left(\left|\frac{d E_{\text {mag }}}{d t}\right|\right)^{-1} \sim \frac{L}{4 V_{\text {in }}}
$$

and this should be the timescale of the flare. Using this timescale, we can estimate the inflow velocity $V_{\text {in }}$ as

$$
V_{\text {in }} \sim \frac{L}{4 \tau_{\text {flare }}} .
$$


To evaluate the reconnection rate in non-dimensional form $M_{\mathrm{A}} \equiv V_{\text {in }} / V_{\mathrm{A}}$, we must estimate the Alfvén velocity in the inflow region $V_{\mathrm{A}}=B_{\mathrm{cor}} / \sqrt{4 \pi \rho}$. Hence, if we measure the coronal density $\rho$, the spatial scale of the flare $L$, the magnetic flux density in the corona $B_{\text {cor }}$, and the timescale of flares $\tau_{\text {flare }}$, we can calculate inflow velocity $V_{\text {in }}$, Alfvén velocity $V_{\mathrm{A}}$, and reconnection rate $M_{\mathrm{A}}$.

\subsection{How to Determine the Physical Parameters of the Solar Flare}

In this subsection we describe how to determine the parameters required for derivation of the inflow velocity $V_{\text {in }}$ and the Alfvén velocity $V_{\mathrm{A}}$. To estimate the inflow velocity $V_{\text {in }}$ by using equation (4), we need the spatial and temporal scales of each flare: $L$ and $\tau_{\text {flare }}$. First, the flare size $L$ is determined by soft X-ray images taken by the Soft X-ray Telescope (SXT; Tsuneta et al. 1991) onboard the Yohkoh satellite (Ogawara et al. 1991) with the beryllium (Be) filter. We define $L$ as the square root of the area where the intensity is more than $10 \%$ of the maximum value in each frame and it is measured at the time when GOES X-ray intensity attains its maximum value in each event. The error of $L$ is considered as the difference in definition of the size; the minimum size is defined by the area where the intensity is more than $30 \%$ of the maximum value in each frame and the maximum size is defined by the area where the intensity is more than $3 \%$ of the maximum value. The timescale of a flare $\tau_{\text {flare }}$ is defined as the period from the onset to the peak of GOES X-ray flux ${ }^{1}$. Since we use one-min average of GOES X-ray flux data, the error of this timescale is considered as one minute. In equations (1) and (2), the spatial scale of the flare $L$ should be the length of the current sheet. Although we cannot measure it or estimate the ratio of the current sheet to the global size accurately, we can consider the length of the current sheet is the same order as the size of system since the flare size $L$ is measured in the impulsive phase.

To estimate the Alfvén velocity in the inflow region $V_{\mathrm{A}}$, we need coronal density $\rho$ and coronal magnetic flux density $B_{\text {cor }}$. Owing to difficulty in measuring the coronal density $\rho$, we assume it $1.67 \times 10^{-15} \mathrm{~g} \mathrm{~cm}^{-3}$ that is the typical value in the active region. The coronal magnetic flux density $B_{\text {cor }}$ is also difficult to measure directly. Therefore, we try to evaluate it in two ways. In the first method (method 1), we use the photospheric magnetograms from the Michelson Doppler Imager (MDI; Scherrer et al. 1995) onboard the Solar and Heliospheric Observatory (SOHO; Domingo et al. 1995). We define the representative magnetic flux density for each flare as follows: First, using soft X-ray images taken by SXT with the Be filter, we determine the area in which the intensity is larger than a threshold value. Second,

\footnotetext{
${ }^{1}$ http://www.ngdc.noaa.gov/stp/SOLAR/ftpsolarflares.html
} 
we measure the magnetic flux in the area and obtain the average of unsigned magnetic flux density. Changing this threshold value from $10 \%$ to $50 \%$ of the maximum soft X-ray intensity of each image, magnetic flux densities are obtained as a function of the threshold value of the soft X-ray intensity. Finally, we define representative magnetic flux density $B_{\mathrm{ph}}$ as the midpoint of the maximum and the minimum of the obtained flux density in this range and consider the extent from the maximum to the minimum as the error range of $B_{\mathrm{ph}}$.

In Figure 1 we show the result of the GOES M3.9 class flare on January 18 as an example. The left panel of Figure 1 illustrates the photospheric magnetogram of the flare region. White and black indicate positive and negative polarities in the magnetogram. The overlaid contours correspond to 10,30 , and $50 \%$ of the maximum soft X-ray intensity. The right panel of Figure 1 shows photospheric magnetic flux density as a function of the threshold value of the soft X-ray intensity. The dotted lines indicate the maximum and minimum flux density and the solid line corresponds to the midpoint value, which is defined as the representative photospheric flux density of this event.

In order to obtain the coronal magnetic flux density, we assume

$$
B_{\mathrm{cor}}=\alpha_{B} B_{\mathrm{ph}}
$$

and

$$
\alpha_{B}=\text { const. }=0.3
$$

among all flares. The validity of this assumption will be discussed in $§ 4.1$.

The second method (method 2) to obtain the coronal flux density is as follows. If all the magnetic energy stored in the corona before the reconnection process turns into the thermal energy in the reconnected loop, we obtain

$$
p_{\text {loop }} \sim \frac{B_{\text {eq }}^{2}}{8 \pi},
$$

where $p_{\text {loop }}$ is the plasma pressure in the flare loop and $B_{\text {eq }}$ is the magnetic flux density in the corona. To distinguish this magnetic flux density from that derived by method 1 , we use the subscript "eq". The value of $p_{\text {loop }}$ is estimated from the color temperature $T$ and the emission measure EM calculated from the GOES X-ray data (Garcia 1994), that is,

$$
p_{\text {loop }}=n_{\text {loop }} k_{\mathrm{B}} T
$$

where

$$
n_{\text {loop }} \sim 2 \sqrt{\frac{E M}{L^{3}}},
$$

$n_{\text {loop }}$ is the number density in the flare loop, and $k_{\mathrm{B}}$ is the Boltzmann constant. Since the GOES X-ray flux data have no spatial resolution, we use the flare size measured in 
the SXT image as the size $L$ in equation (9). Hence, we can estimate $B_{\text {eq }}$ and assume $B_{\text {cor }} \sim B_{\text {eq }}$. The error of this flux density $B_{\text {eq }}$ results from the error in deriving temperature and emission measure from the GOES X-ray data. On the basis of the description in Garcia (1994), estimated uncertainty in the derived temperature depends on the temperature and is calculated at $13 \%$ around $10 \mathrm{MK}$. We calculate this uncertainty as a function of temperature and then obtain the propagated error of $B_{\text {eq }}$.

\subsection{Event Selection}

As we describe in the previous subsection, we use SXT images, MDI magnetograms, and GOES X-ray flux data. We survey flares that occurred in 2000 under the following criteria: (1) GOES class of the flare is above C6.0, (2) the flare site is included in the field of view of the partial-frame SXT image with the Be filter taken at the peak of the GOES soft X-ray flux, and (3) the distance from the disk center to the flare region is within 800 arcsecs to avoid the line-of-sight effect in estimation of the magnetic flux density. From the flare list obtained by GOES data, 482 flares meet the above criterion (1) and 77 of those flares satisfy all the criteria.

\section{RESULT}

Using the method described in the previous section, we have analyzed 77 flares occurred in the year of 2000. First, we examine dependence of physical parameters of these flares on GOES class. In Figure 2 we plot the parameters of flares as a function of GOES class. Temporal scale $\tau_{\text {flare}}$, spatial scale $L$, photospheric field $B_{\mathrm{ph}}$ obtained by method 1 , and coronal field $B_{\text {eq }}$ obtained by method 2 are shown in Figure 2a, 2b, 2c, and 2d, respectively. We find from these plots: (1) There is weak correlation between the timescale $\tau_{\text {flare }}$ and the GOES peak flux (Panel a). This tendency is consistent with Figure 3 of Veronig et al. (2002) and Figure 2 of Kay et al. (2003). (2) The characteristic size of flares $L$ shows a larger scatter when the GOES peak flux is smaller (Panel b). Garcia (1998) reported the relation between the GOES peak flux and the loop length of flares in their Figure 5. Comparing our results

with theirs, the value of the size is in the same range, although clear correlation between these parameters cannot be found out in their results. (3) There is a threshold value in the magnetic flux density $\left(B_{\mathrm{ph}}\right.$ and $\left.B_{\text {eq }}\right)$ that increases as the GOES peak flux increases (Panels c and d). For example, the X-class flares occurred only when $B_{\text {ph }}$ was larger than $\approx 100$ Gauss. 
Second, we examine the relationship between the physical parameters of flares. Figure 3 shows the spatial scale $L$ plotted against the timescale $\tau_{\text {flare }}$ and tells that the spatial scale $L$ tends to be larger with increasing timescale. This result is consistent with the result shown in Figure 1c of Garcia (1998), although the tendency is not so clear in their figure due to a small number of their analyzed events. The lines in Figure 3 indicate the inflow velocity $V_{\text {in }}$ given by equation (4). The inflow velocities are found to be distributed from a few $\mathrm{km} \mathrm{s}^{-1}$ to several tens $\mathrm{km} \mathrm{s}^{-1}$. These inflow velocities are comparable to those in the previous studies. Detailed discussion is given in $\S 4.2$. The left panel of Figure 4 shows the photospheric flux density $B_{\mathrm{ph}}$ obtained by method 1 plotted against the timescale $\tau_{\text {flare }}$, and in the right panel of Figure 4 the coronal flux density $B_{\text {eq }}$ obtained by method 2 is plotted against the timescale $\tau_{\text {flare }}$. Assuming the ratio of coronal flux density to photospheric flux density $\alpha_{B} \equiv B_{\text {cor }} / B_{\mathrm{ph}}$ is 0.3 and the coronal density $\rho$ is $1.67 \times 10^{-15} \mathrm{~g} \mathrm{~cm}^{-3}$, the values of magnetic flux density in the left panel of Figure 4 correspond to $10^{3}-10^{4} \mathrm{~km} \mathrm{~s}^{-1}$ of the Alfvén velocity in the inflow region $V_{\mathrm{A}}$. The values of $B_{\text {eq }}$ in the right panel of Figure 4 also correspond to the same range of $V_{\mathrm{A}}$.

Using these results of $V_{\text {in }}$ and $V_{\mathrm{A}}$, we examine the reconnection rate $M_{\mathrm{A}}$. In the left panel of Figure 5, the reconnection rate obtained by method 1 is plotted against the GOES class. The values of reconnection rate are distributed around $10^{-3}-10^{-2}$. Note again that we assume $\alpha_{B} \equiv B_{\text {cor }} / B_{\mathrm{ph}}=0.3$ in this plot. By changing $\alpha_{B}$, the data points will shift proportionally to the value. The right panel of Figure 5 shows the reconnection rate calculated by using the magnetic flux density $B_{\text {eq }}$ obtained by method 2 against the GOES class of each flare. The reconnection rates are also distributed around $10^{-3}-10^{-2}$. Both panels of Figure 5 show that the reconnection rate tends to diminish as the GOES class of the flare increases.

As mentioned in $\S 2.2$, we estimate the coronal magnetic flux density in two ways. In Figures $2 \mathrm{c}$ and $2 \mathrm{~d}$, although the flux density obtained by method 1 (Figure $2 \mathrm{c}$ ) appears more broadly scattered than that obtained by method 2 (Figure $2 \mathrm{~d}$ ), the values of flux density show the similar distribution. Moreover, the magnetic flux density obtained by these two methods has a similar distribution as a function of $\tau_{\text {flare }}$ (Figure 4). Two panels of Figure 5 show that the values of the reconnection rate obtained by methods 1 and 2 are the same range and display the same dependence on the GOES class. Hence, we conclude that the results by these two methods are consistent with each other. 


\section{DISCUSSION}

\subsection{Ratio of Coronal Flux Density to Photospheric Flux Density}

We must examine whether the assumption that the ratio of coronal flux density to photospheric flux density $\alpha_{B} \equiv B_{\text {cor }} / B_{\text {ph }}$ equals 0.3 is appropriate one. In Figure 6 , we compare the photospheric flux density $B_{\text {ph }}$ obtained by method 1 with the equipartitional flux density $B_{\text {eq }}$ obtained by method 2 . The lines in the figure correspond to $0.1,0.3$, and 1 of $B_{\text {eq }} / B_{\mathrm{ph}}$. This figure shows the ratios are distributed around 0.3 , and if we consider $B_{\text {eq }}$ as the coronal flux density, our assumption $\alpha_{B}=0.3$ is not inappropriate.

Dere (1996) estimated coronal flux density from data of photospheric flux density described in the scientific literature. He calculated typical magnetic flux density as a function of height as photospheric magnetogram forms the base. The formula for his analyzed active region observed on 1974 January 16 is

$$
B(h)=\left[45 \exp \left(\frac{-h}{3.5 \times 10^{8} \mathrm{~cm}}\right)+50 \exp \left(\frac{-h}{3.0 \times 10^{9} \mathrm{~cm}}\right)\right] \mathrm{G},
$$

where $h$ is height above the photosphere. He applied this formula to calculate the coronal flux density of all his analyzed events in active regions. Using the typical spatial scale of flare loops in our analysis $L \sim 10^{9} \mathrm{~cm}$ as the height $h$, we obtain the coronal field of 38 $\mathrm{G}$ by equation (10); we can also calculate the photospheric flux density of his model at 95 $\mathrm{G}$ by taking $h=0$ in equation (10). Therefore the ratio of the coronal flux density to the photospheric flux density is roughly estimated at 0.4 . This formula probably underestimates the magnetic flux density, because the photospheric flux density analyzed in our paper is in the range from $100 \mathrm{G}$ to $700 \mathrm{G}$. Hence, we also did a calculation of the coronal potential field for some particular events for more investigations. The left panel of Figure 7 shows average potential magnetic field strength of X2.3 flare occurred on 2000 November 24. This flux density is extrapolated by using the software package MAGPACK2 (Sakurai 1982) and its strength is determined and averaged in the central portion of the flare. In this calculation, the coronal flux density at the height of $2.56 \times 10^{9} \mathrm{~cm}$ that corresponds to the flare size of this event is $83 \mathrm{G}$. As shown in the left panel of Figure 7, the photospheric field strength is $335 \mathrm{G}$ at $h=0$. Note, however, that $\alpha_{B}$ in equation (5) should be calculated by the ratio of the coronal flux density to the line-of-sight strength of the photospheric field. Therefore we calculate the line-of-sight component of photospheric flux density using calculation of the potential field; the spatial average of the line-of-sight component at $h=0$ in the flare site is $B_{\mathrm{ph}}=151 \mathrm{G}$, which is shown as an asterisk in the left panel of Figure 7. Accordingly, we obtain the ratio of coronal flux density to the line-of-sight component of photospheric flux density $\alpha_{B}$ is nearly 0.6. In the right panel of Figure 7, we show the average potential 
magnetic field strength of M3.7 flare occurred on 2000 July 14 in the same way. Concerning this event, the photospheric flux density is $214 \mathrm{G}$ at $h=0$, the coronal flux density at the height of the flare loop is $22 \mathrm{G}$ and the average line-of-sight component of photospheric field is $B_{\mathrm{ph}}=119 \mathrm{G} ; \alpha_{B}$ is roughly 0.2 . We calculate some other events and obtain the ratio $\alpha_{B}$ in the range of $0.2-0.6$.

Considering these estimates of the ratio, the assumption $\alpha_{B} \equiv B_{\text {cor }} / B_{\mathrm{ph}} \sim 0.3$ is probably plausible. As a result, on the basis of the result described in $\S 3$, we find the reconnection rate $M_{\mathrm{A}}$ is $10^{-3}$ for our analyzed events.

\subsection{Comparison with the Previous Study}

In this section, we compare our results with the previous studies to evaluate our results. Using inflow pattern in extreme ultraviolet images, the inflow velocity $V_{\text {in }}$ was measured around $5 \mathrm{~km} \mathrm{~s}^{-1}$ by Yokoyama et al. (2001), while Narukage \& Shibata (2006) obtained 2.6 $-38 \mathrm{~km} \mathrm{~s}^{-1}$ in six events. Including other indirect measurements, the inflow velocity is in the same range as our results (Figure 3). Moreover, we analyze one of Narukage \& Shibata's events on 1999 May 27 using our method described in $§ 2.2$. We estimate the inflow velocity at $5.2 \mathrm{~km} \mathrm{~s}^{-1}$, while they reported the inflow velocity of this event is $12.5-14.9 \mathrm{~km} \mathrm{~s}^{-1}$ or $31.3-37.3 \mathrm{~km} \mathrm{~s}^{-1}$. The former is obtained by the method based on Yokoyama et al. (2001), while the latter is obtained by the estimation based on Chen et al. (2004). In either case, the velocity obtained by our method is less than that obtained by their method.

Next, we compare our results with those of Isobe et al. (2005), since some of our analyzed events are also examined by them. In our statistical study we use relatively simple methods to study a large number of events, while they dealt with three events in detail in their case study. Table 1 summarizes parameters of the flares obtained in this work and in Isobe et al. (2005), mainly referring to their Table 1 . We calculate some parameters based on this table as follows. Isobe et al. (2005) measured the width $L_{x}$, the length $L_{y}$, and the height $L_{z}$ of the flare arcade separately. To compare with our measurement of $L$, we calculate the flare size $L$ is defined as the cubic root of the product of $L_{x}, L_{y}$, and $L_{z}$ given in the paper. The averaged energy release rate $E_{\text {flare }} / \tau$ is defined as the thermal energy in the flare loop divided by the temporal scale,

$$
E_{\text {flare }} / \tau \equiv \frac{3}{2} n_{\text {loop }} k_{\mathrm{B}} T L^{3} / \tau_{\text {flare }} .
$$

This value is calculated from the peak value of $E_{\text {th }}$ in Isobe et al.'s Figures 6,8 , and 10 together with our GOES timescale $\tau_{\text {flare }}$. Moreover, we define the energy release rate $\left|d E_{\mathrm{mag}} / d t\right|$ as equation (2) for our study and as $H$ in Isobe et al. (2005) for their study, both of which 
correspond to the Poynting flux into the diffusion region. Furthermore it should be noted here that the GOES C-class event in Table 1 is not one of our analyzed events in this statistical study due to lack of the non-saturated SXT image with the Be filter at the flare peak. Therefore using the last non-saturated image with the Be filter taken at three minutes before the peak, we calculate the parameters in this table for only comparison.

Comparing our result with Isobe et al. (2005), we find that in our study the inflow velocity $V_{\text {in }}$ is systematically smaller. Since we calculate $V_{\text {in }}$ as $L /\left(4 \tau_{\text {flare }}\right), V_{\text {in }}$ obtained in our study is an average value in the impulsive phase of a flare. On the other hand, Isobe et al. (2005) estimated $V_{\text {in }}$ using the peak value of the energy release rate in the impulsive phase of the flares. Therefore our small inflow velocity $V_{\text {in }}$ perhaps reflects nonsteadiness of the reconnection rate in the reconnection process. Moreover, owing to our larger coronal magnetic flux density $B_{\text {cor }}$, the coronal Alfvén velocity $V_{\mathrm{A}}$ of our result is systematically larger than those of their result. Consequently, this larger $V_{\mathrm{A}}$ and smaller $V_{\text {in }}$ make the reconnection rate $M_{\mathrm{A}}$ smaller than a factor of 2 - 10. Nevertheless, considering energy budget of each flare, we note that our result is not inconsistent; the energy release rate $\left|d E_{\mathrm{mag}} / d t\right|$ is comparable with the averaged energy release rate $E_{\text {flare }} / \tau$ during the impulsive phase of the flare.

\subsection{Reconnection Rate and Reconnection Model}

Here we discuss the possible implication to the models from the reconnection rate obtained in this work. As mentioned in $\S 1$, different models show not only different values of the reconnection rate but also different dependence of the reconnection rate on the magnetic Reynolds number $R_{\mathrm{m}}=V_{\mathrm{A}} L / \eta$. Therefore we examine this dependence. The reconnection rate $M_{\mathrm{A}}$ is plotted against the magnetic Reynolds number $R_{\mathrm{m}}$ in Figure 8 . The reconnection rate and the Reynolds number obtained by method 1 is shown in the left panel of the figure and those obtained by method 2 is shown in the right panel. In the left panel, the dash-dotted line indicates the least-squares fitting of the data, which yields $M_{\mathrm{A}} \propto R_{\mathrm{m}}^{-0.8}$, while in the right panel there is no clear dependence since the magnetic Reynolds number is distributed within one order of magnitude. To see the figure clearly, the error bars of the magnetic Reynolds number are omitted in the right panel, though the error is one order of magnitude at most. The dotted lines in both panels indicate the dependence of the reconnection rate on the magnetic Reynolds number that is predicted by the Petschek model and the dashed lines indicate the Sweet-Parker-type dependence $M_{\mathrm{A}} \propto R_{\mathrm{m}}^{-0.5}$. In calculation of the magnetic diffusivity $\eta$ in $R_{\mathrm{m}}$, we assume the resistivity is attributed to the Coulomb collision (Spitzer 1956) and the plasma temperature is $10^{7} \mathrm{~K}$. 
In the process of estimating the reconnection rate $M_{\mathrm{A}}$ and the magnetic Reynolds number $R_{\mathrm{m}}$ shown in Figure 8, we have following assumptions: (1) the coronal density $\rho$ is $1.67 \times 10^{-15} \mathrm{~g} \mathrm{~cm}^{-3}$, (2) the ratio of coronal flux density to photospheric flux density $\alpha_{B} \equiv B_{\text {cor }} / B_{\text {ph }}$ equals 0.3 , and (3) the plasma temperature is $10^{7} \mathrm{~K}$. If the coronal density $\rho$ and/or the ratio $\alpha_{B}$ deviate from the assumed value, the values of the Alfvén velocity and the magnetic Reynolds number are affected. However these values will only shift by some factors on the whole. Therefore the distribution shown in Figure 8, i.e., the power index of the dependence of the reconnection rate $M_{\mathrm{A}}$ on the magnetic Reynolds number $R_{\mathrm{m}}$ will not be greatly affected by assumptions (1) and (2). Moreover, we can consider the factor to shift to be less than one order of magnitude, because the coronal density $\rho$ is thought to be within at most one order of magnitude from the typical value and the ratio $\alpha_{B}$ is estimated in the range of $0.2-0.6$ as mentioned in $\S 4.1$. Furthermore, in analyzing by method 2, we calculate the plasma temperature from GOES X-ray data and find that the observed temperature of plasma is distributed within less than one order of magnitude around $10^{7} \mathrm{~K}$. Hence assumption (3) is thought to have little effect on our results.

From the comparison of ours with the previous results mentioned in $\S 4.2$ (e.g., Isobe et al. 2005; Narukage \& Shibata 2006), the error of the obtained reconnection rate shown in our figures is probably a little underestimated. Besides the assumptions mentioned in the previous paragraph, other uncertainties, such as the uncertainties in the measurements of the size $L$, the timescale $\tau_{\text {flare }}$, the temperature $T$ and the emission measure $E M$, will affect our result. Even after considering all these effects, the value of $M_{\mathrm{A}}$ in Figure 8 is found within one order of magnitude from the theoretical value of the Petschek model, while dependence of the reconnection rate on the magnetic Reynolds number tends to be stronger than that in the Petschek model. This result suggests two possibilities: One is the occurrence of the Petschek-type reconnection in the flares and the other is the SweetParker model with the magnetic diffusivity $\eta$ enlarged by some diffusion processes, such as the MHD turbulence. Owing to the uncertainties, we cannot immediately rule out the possibility of either the Petschek model or the generalized Sweet-Parker model from our result. We expect to discriminate between these two interpretations by the observations of Solar-B. If the Sweet-Parker reconnection with this enhanced resistivity occurs in a flare, the thickness of the diffusion region is estimated to be $100 \mathrm{~km}$, when the length of the diffusion region is approximately the size of flare $L \sim 10^{9} \mathrm{~cm}$ and the reconnection rate is $M_{\mathrm{A}} \sim 0.01$. Because the size of diffusion region is less than the limit of resolution, the structure of turbulence is expected to be observed as a single-pixel line-broadening with Solar-B. On the other hand, if the Petschek-type reconnection occurs, the slow shock structure is expected to be observed. 


\section{CONCLUSION}

We analyze the data of flares occurred in the year of 2000 and measure the temporal scale $\tau$, the size $L$, and the magnetic field $B$ of each flare. First, we examine the dependence of these parameters on the GOES class and find that the sizes of flares tend to be distributed more broadly as the GOES class becomes weaker, and there is a threshold value in both photospheric and coronal magnetic flux density that increases as the GOES peak flux increases. Second, we examine the relationship between those parameters. There is weak correlation between temporal and spatial scales of flares, while there is little correlation between temporal scale and magnetic flux density. Finally, we try to estimate the reconnection rate in each

flare. The values are distributed in the range from $10^{-3}$ to $10^{-2}$ and the reconnection rate decreases as the GOES class increases. The value of the reconnection rate obtained in our study is within one order of magnitude from the predicted maximum value of the Petschek model, although the dependence of the reconnection rate on the magnetic Reynolds number tends to be stronger than that in the Petschek model.

We thank H. Isobe and T. J. Okamoto for careful reading and helpful comments. We also thank K. Shibata for useful discussions. We are grateful to T. Sakurai for allowing us to use his potential field program (MAGPACK2). Data analysis were carried out on the computer system at the Nobeyama Solar Radio Observatory of the National Astronomical Observatory of Japan and the Solar Data Archive (SODA) analysis server at the PLAIN center, ISAS/JAXA. The Yohkoh satellite is a Japanese national project, launched and operated by ISAS/JAXA, and involving many domestic institutions, with international collaboration with the US and the UK. Geostationary Operational Environmental Satellites (GOES) is operated by the National Oceanic and Atmospheric Administration's (NOAA's) National Environmental Satellite, Data, and Information Service (NESDIS). SOHO is a project operated by the European Space Agency and the US National Aeronautics and Space Administration.

\section{REFERENCES}

Chen, P. F., Shibata, K., Brooks, D. H., \& Isobe, H. 2004, ApJ, 602, L61

Dere, K. P. 1996, ApJ, 472, 864

Domingo, V., Fleck, B., \& Poland, A. I. 1995, Sol. Phys., 162, 1

Garcia, H. A. 1994, Sol. Phys., 154, 275

-. 1998, ApJ, 504, 1051 
Isobe, H., Morimoto, T., Eto, S., Narukage, N., \& Shibata, K. 2002a, in Multi-Wavelength Observations of Coronal Structure and Dynamics, ed. P. C. H. Martens and D. P. Cauffman (Oxford: Pergamon), 171

Isobe, H., Takasaki, H., \& Shibata, K. 2005, ApJ, 632, 1184

Isobe, H., Yokoyama, T., Shimojo, M., Morimoto, T., Kozu, H., Eto, S., Narukage, N., \& Shibata, K. 2002b, ApJ, 566, 528

Kay, H. R. M., Harra, L. K., Matthews, S. A., Culhane, J. L., \& Green, L. M. 2003, A\&A, 400, 779

Lin, J., Ko, Y. K., Sui, L., Raymond, J. C., Stenborg, G. A., Jiang, Y., Zhao, S., \& Mancuso, S. 2005, ApJ, 622, 1251

Matthaeus, W. H., \& Lamkin, S. L. 1985, Phys. Fluids, 28, 303

Narukage, N., \& Shibata, K. 2006, ApJ, 637, 1122

Nitta, S. 2004, ApJ, 610, 1117

Ogawara, Y., Takano, T., Kato, T., Kosugi, T., Tsuneta, S., Watanabe, T., Kondo, I., \& Uchida, Y. 1991, Sol. Phys., 136, 1

Ohyama, M., \& Shibata, K. 1997, PASJ, 49, 249

-. 1998, ApJ, 499, 934

Parker, E. N. 1957, J. Geophys. Res., 62, 509

Petschek, H. E. 1964, in The Physics of Solar Flares, ed. W. N. Hess., 425

Sakurai, T. 1982, Sol. Phys., 76, 301

Scherrer, P. H., Bogart, R. S., Bush, R. I., Hoeksema, J. T., Kosovichev, A. G., Schou, J., Rosenberg, W., Springer, L., Tarbell, T. D., Title, A., Wolfson, C. J., Zayer, I., \& MDI Engineering Team. 1995, Sol. Phys., 162, 129

Shibata, K., \& Tanuma, S. 2001, Earth, Planets, and Space, 53, 473

Spitzer, L. 1956, Physics of Fully Ionized Gases (New York: Interscience Publishers)

Sweet, P. A. 1958, in IAU Symposium no. 6, Electromagnetic Phenomena in Cosmical Physics, ed. B. Lehnert (Cambridge University Press), 123 
Tajima, T., \& Shibata, K. 1997, Plasma Astrophysics (Reading, Mass. :Addison- Wesley)

Tsuneta, S. 1996, ApJ, 456, 840

Tsuneta, S., Acton, L., Bruner, M., Lemen, J., Brown, W., Caravalho, R., Catura, R., Freeland, S., Jurcevich, B., \& Owens, J. 1991, Sol. Phys., 136, 37

Tsuneta, S., Masuda, S., Kosugi, T., \& Sato, J. 1997, ApJ, 478, 787

Veronig, A., Temmer, M., Hanslmeier, A., Otruba, W., \& Messerotti, M. 2002, A\&A, 382, 1070

Yokoyama, T., Akita, K., Morimoto, T., Inoue, K., \& Newmark, J. 2001, ApJ, 546, L69

This preprint was prepared with the AAS IATEX macros v5.2. 
Table 1. Parameters of the Flares

\begin{tabular}{|c|c|c|c|c|c|c|c|c|c|}
\hline \multirow[t]{3}{*}{ Parameter } & \multicolumn{3}{|c|}{2000 Nov 24 15:13 X2.3 flare } & \multicolumn{3}{|c|}{ 2000 Jul 14 13:52 M3.7 flare } & \multicolumn{3}{|c|}{2000 Nov 16 0:40 C8.9 flare } \\
\hline & \multicolumn{2}{|c|}{ This Work } & \multirow{2}{*}{ Isobe et al. (2005) } & \multicolumn{2}{|c|}{ This Work } & \multirow[t]{2}{*}{ Isobe et al. (2005) } & \multicolumn{2}{|c|}{ This Work } & \multirow[t]{2}{*}{ Isobe et al. (2005) } \\
\hline & Method 1 & Method 2 & & Method 1 & Method 2 & & Method 1 & Method 2 & \\
\hline$\tau(\mathrm{sec})$ & \multicolumn{2}{|c|}{1320} & & \multicolumn{2}{|c|}{480} & & \multicolumn{2}{|c|}{1200} & \\
\hline$B_{\mathrm{ph}}(\mathrm{G})$ & \multicolumn{2}{|c|}{$205 \pm 17$} & 449 & \multicolumn{2}{|c|}{$106 \pm 11$} & 117 & \multicolumn{2}{|c|}{$30 \pm 4$} & 106 \\
\hline$B_{\text {cor }}(\mathrm{G})$ & 62 & 116 & 41 & 32 & 60 & 44 & 9 & 32 & 11 \\
\hline$L\left(10^{9} \mathrm{~cm}\right)$ & \multicolumn{2}{|c|}{2.56} & 2.8 & \multicolumn{2}{|c|}{2.94} & 2.3 & \multicolumn{2}{|c|}{4.12} & 4.0 \\
\hline$T\left(10^{6} \mathrm{~K}\right)$ & \multicolumn{2}{|c|}{14} & 10 & \multicolumn{2}{|c|}{8.8} & 8.0 & \multicolumn{2}{|c|}{7.2} & 8.0 \\
\hline$V_{\mathrm{in}}\left(\mathrm{cm} \mathrm{s}^{-1}\right)$ & \multicolumn{2}{|c|}{$4.8 \times 10^{5}$} & $1.3 \times 10^{7}$ & \multicolumn{2}{|c|}{$1.5 \times 10^{6}$} & $3.2 \times 10^{6}$ & \multicolumn{2}{|c|}{$8.6 \times 10^{5}$} & $6.7 \times 10^{6}$ \\
\hline$V_{\mathrm{A}}\left(\mathrm{cm} \mathrm{s}^{-1}\right)$ & $4.25 \times 10^{8}$ & $8.0 \times 10^{8}$ & $2.8 \times 10^{8}$ & $2.2 \times 10^{8}$ & $4.2 \times 10^{8}$ & $2.1 \times 10^{8}$ & $6.2 \times 10^{7}$ & $2.3 \times 10^{8}$ & $9.4 \times 10^{7}$ \\
\hline$M_{\mathrm{A}}$ & $1.1 \times 10^{-3}$ & $6.0 \times 10^{-4}$ & $4.7 \times 10^{-2}$ & $7.0 \times 10^{-3}$ & $3.7 \times 10^{-3}$ & $1.5 \times 10^{-2}$ & $1.4 \times 10^{-2}$ & $3.9 \times 10^{-3}$ & $7.1 \times 10^{-2}$ \\
\hline$E_{\text {flare }} / \tau\left(\mathrm{erg} \mathrm{s}^{-1}\right)$ & \multicolumn{2}{|c|}{$1 \times 10^{28}$} & $4 \times 10^{27}$ & \multicolumn{2}{|c|}{$1 \times 10^{28}$} & $3 \times 10^{27}$ & \multicolumn{2}{|c|}{$4 \times 10^{27}$} & $8 \times 10^{26}$ \\
\hline$\left|d E_{\mathrm{mag}} / d t\right|\left(\mathrm{erg} \mathrm{s}^{-1}\right)$ & $2 \times 10^{27}$ & $7 \times 10^{27}$ & $2.7 \times 10^{28 \mathrm{a}}$ & $2 \times 10^{27}$ & $8 \times 10^{27}$ & $6.4 \times 10^{27 \mathrm{a}}$ & $2 \times 10^{26}$ & $2 \times 10^{27}$ & $1.9 \times 10^{27 \mathrm{a}}$ \\
\hline
\end{tabular}

${ }^{\text {a }}$ Variable $H$ in Isobe et al. (2005) 

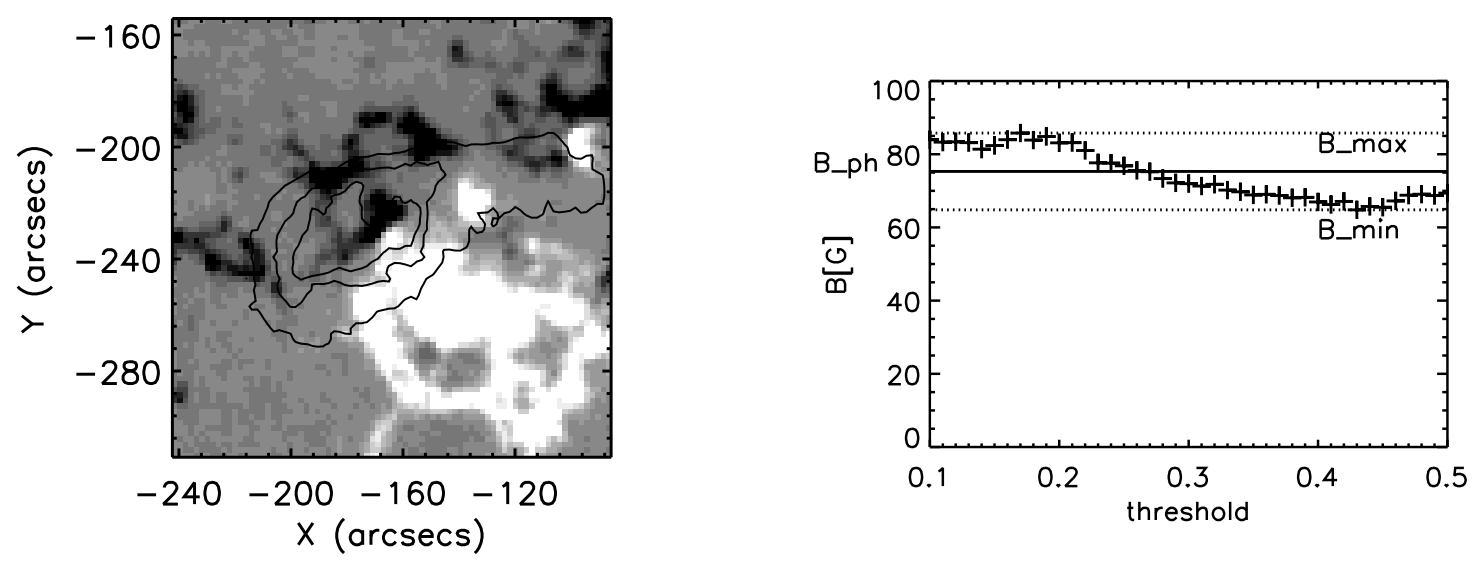

Fig. 1. - Left panel: Soft X-ray contours overlaid on the SOHO/MDI magnetogram of the M3.9 flare on 2000 January 18. The contours show Yohkoh/SXT image; 10, 30, and 50\% of the maximum soft X-ray intensity. White and black indicate positive and negative polarities in the magnetogram. Right panel: Photospheric flux density plotted against threshold value (see text). The dotted lines indicate the maximum and minimum of the flux density and the solid line corresponds to the midpoint of the maximum and the minimum. We define this midpoint as the representative photospheric flux density of this event and the error range as the extent from the minimum to the maximum. 

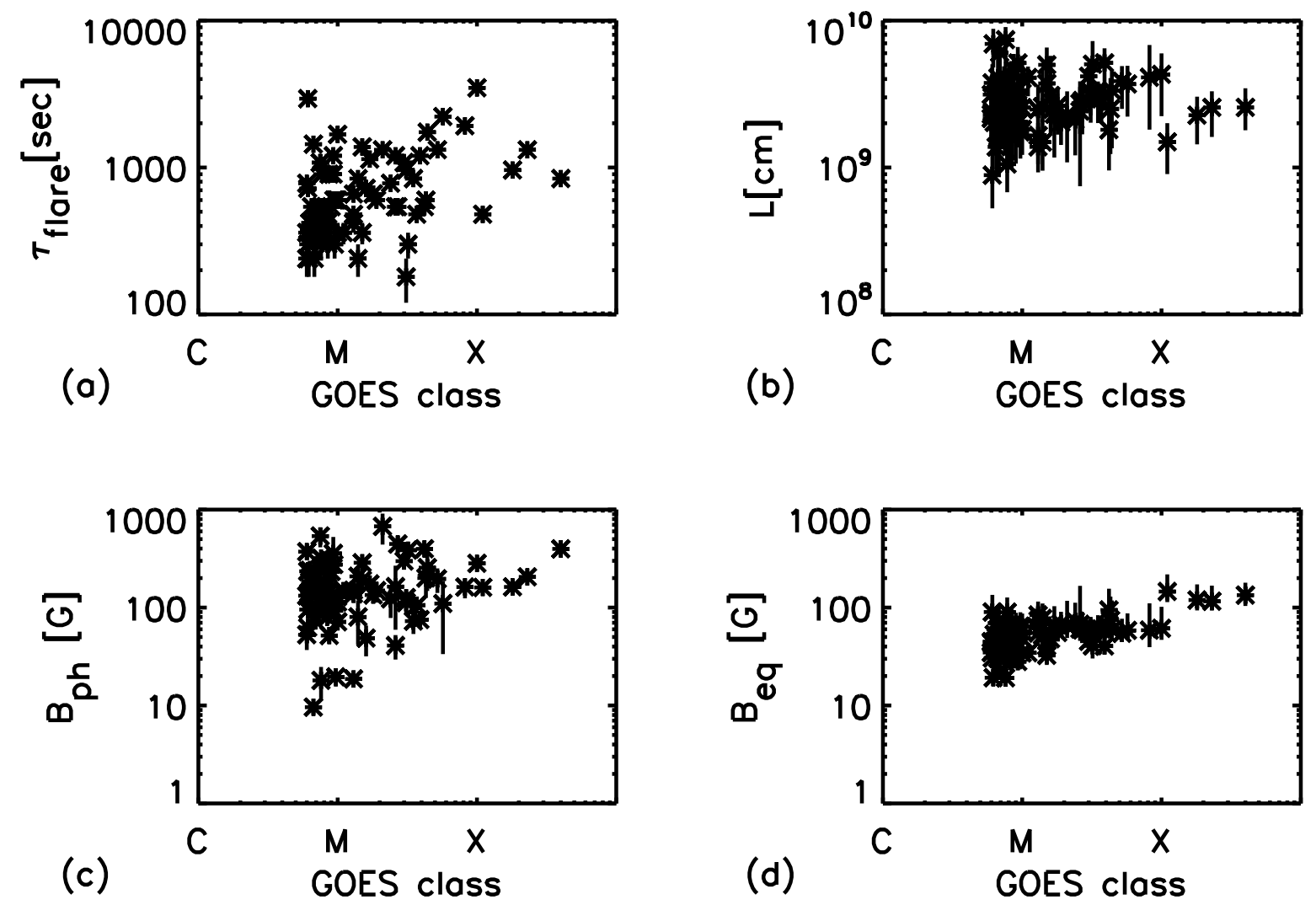

Fig. 2.- Physical parameters of each flare plotted against the GOES class. (a) Time scale $\tau_{\text {flare }}$. (b) Size $L$. (c) Photospheric magnetic flux density $B_{\mathrm{ph}}$ obtained by method 1. (d) Coronal flux density $B_{\text {eq }}$ obtained by method 2 . 


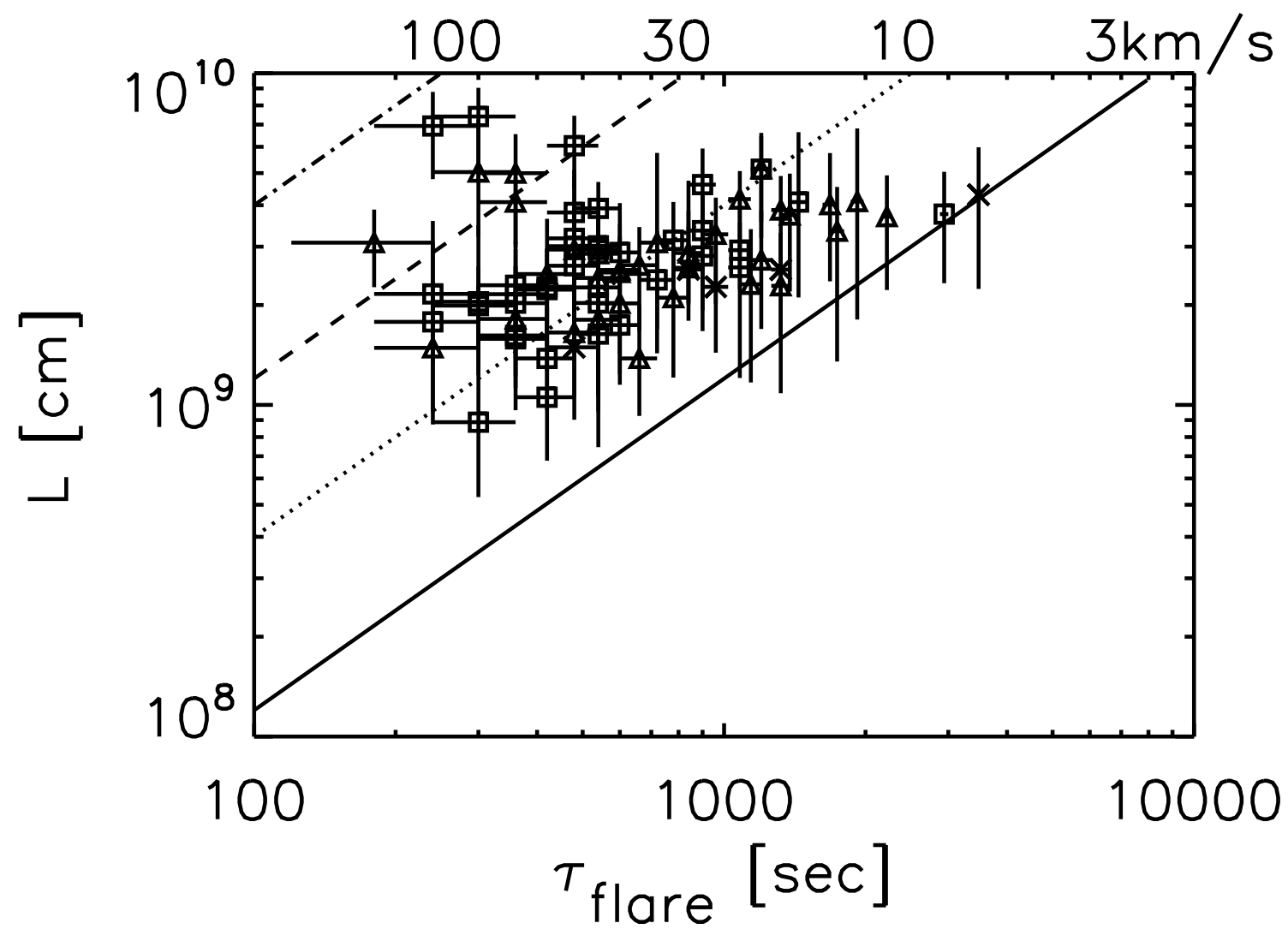

Fig. 3.- Spatial scale of each event plotted against timescale. Crosses, triangles, and squares indicate X-class, M-class, and C-class, respectively. The solid line, dotted line, dashed line, and dash-dotted line indicate $3,10,30$, and $100 \mathrm{~km} \mathrm{~s}^{-1}$ of the inflow velocity $V_{\text {in }}$, respectively. 

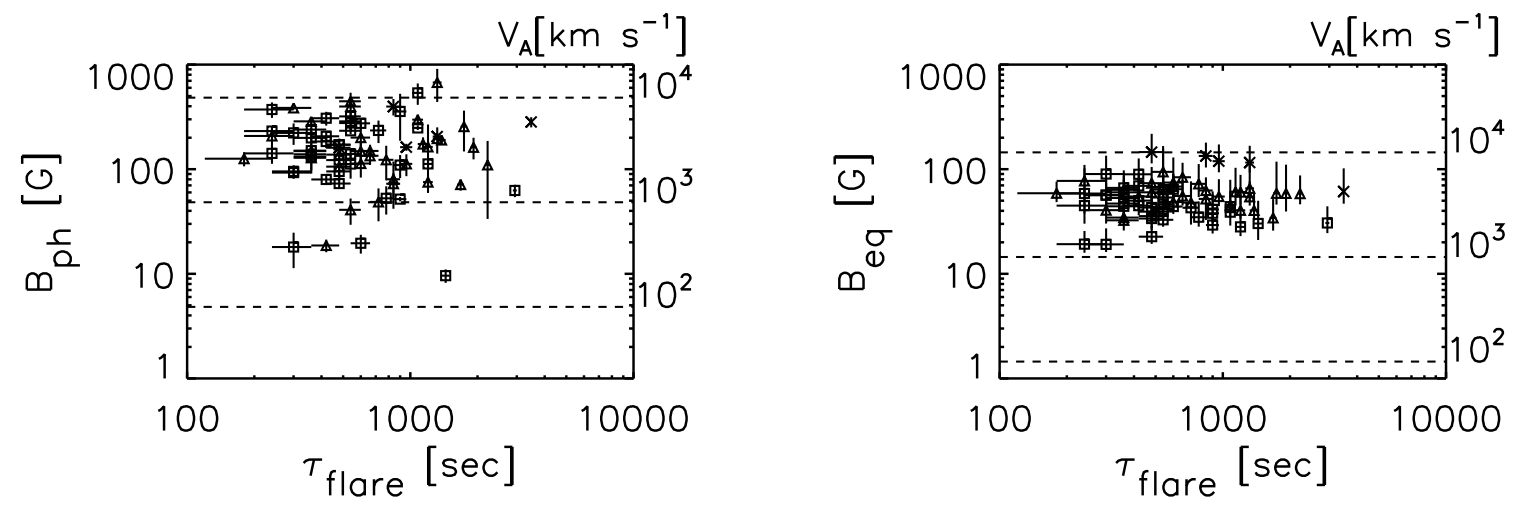

Fig. 4.- Left panel: Photospheric flux density of each event $B_{\mathrm{ph}}$, obtained by method 1 , plotted against timescale of the flares $\tau_{\text {flare }}$. The dashed lines indicate the corresponding coronal Alfvén velocity calculated on the assumption that the coronal density $\rho=1.67 \times$ $10^{-15} \mathrm{~g} \mathrm{~cm}^{-3}$ and $\alpha_{B} \equiv B_{\mathrm{cor}} / B_{\mathrm{ph}}=0.3$. Right panel: Coronal flux density $B_{\text {eq }}$, obtained by method 2, plotted against the timescale $\tau_{\text {flare }}$. The dashed lines indicate the corresponding coronal Alfvén velocity. Meaning of the symbols in these figures is the same as Figure 3.
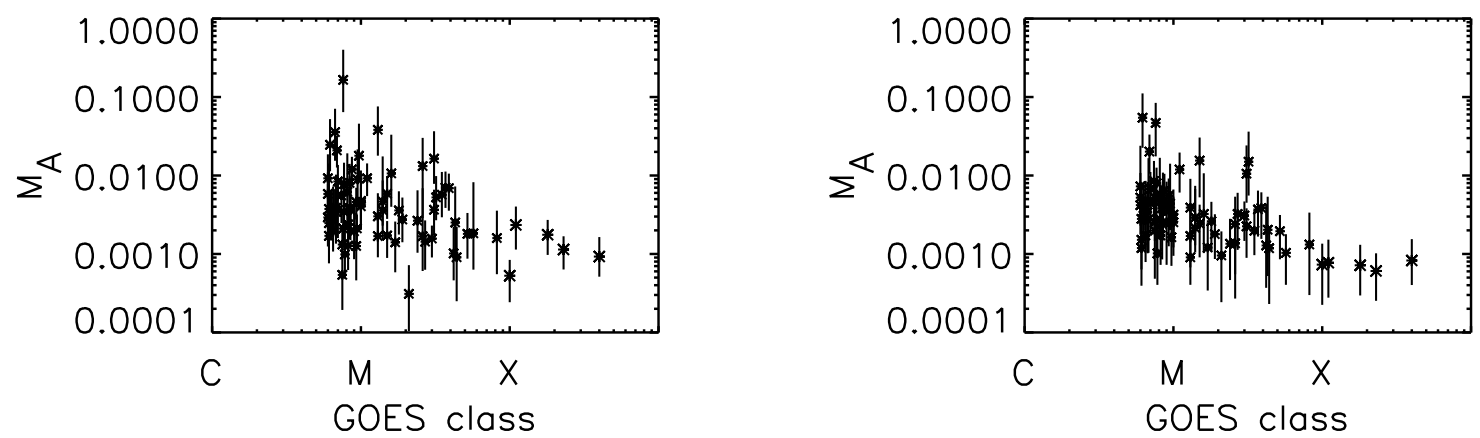

Fig. 5.- Reconnection rate $M_{A}$ plotted against the GOES class of each flare. The left panel shows the reconnection rate obtained by method 1 . We assume $\alpha_{B} \equiv B_{\text {cor }} / B_{\text {ph }}=0.3$ when we calculate $M_{A}$. The right panel shows the reconnection rate obtained by method 2 . 


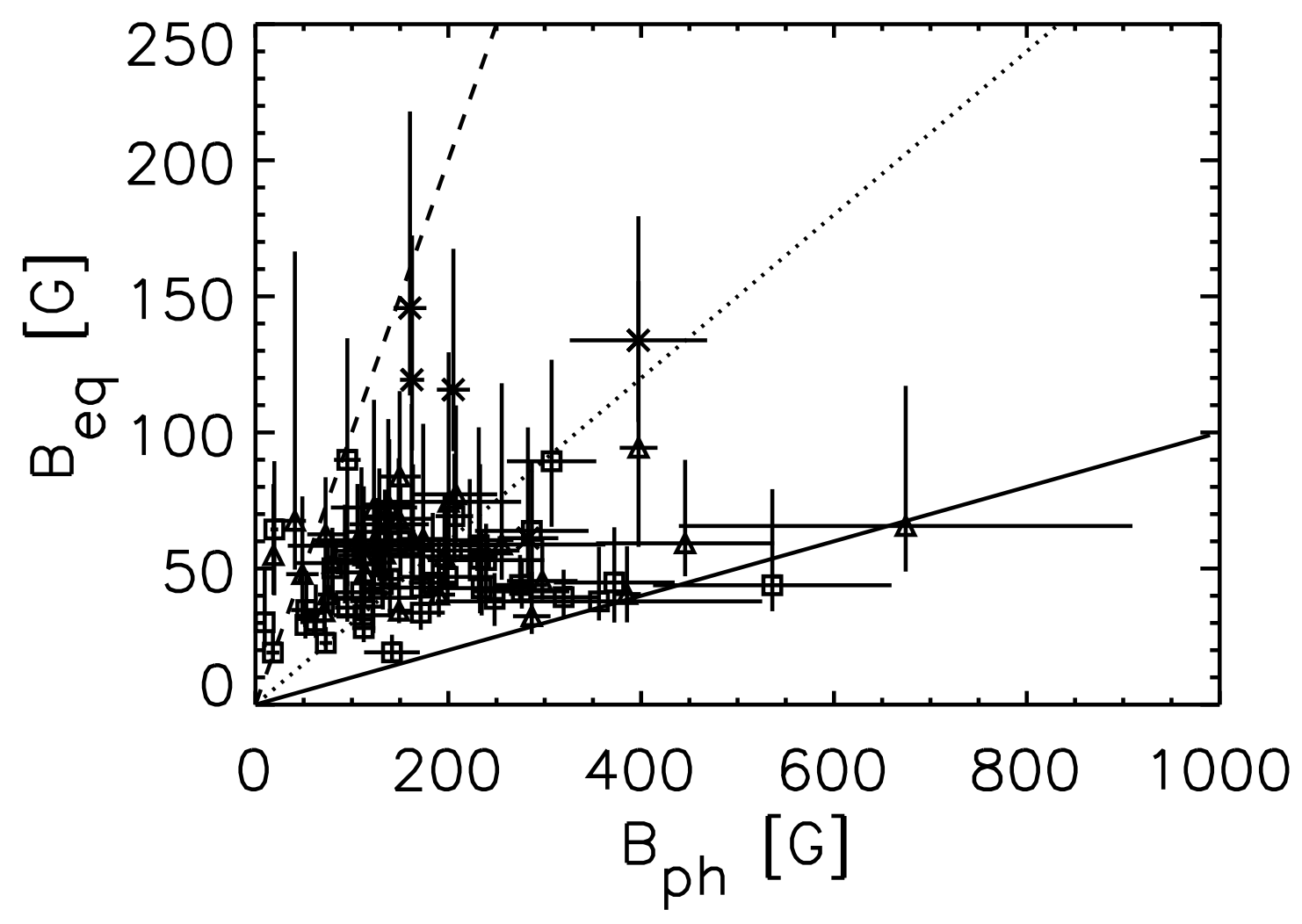

Fig. 6.- Coronal magnetic flux density $B_{\text {eq }}$ plotted against photospheric flux density $B_{\text {ph }}$. The solid line, the dotted line, and the dashed line correspond to $0.1,0.3$, and 1 of $B_{\mathrm{eq}} / B_{\mathrm{ph}}$, respectively. Meaning of the symbols is the same as Figure 3. 

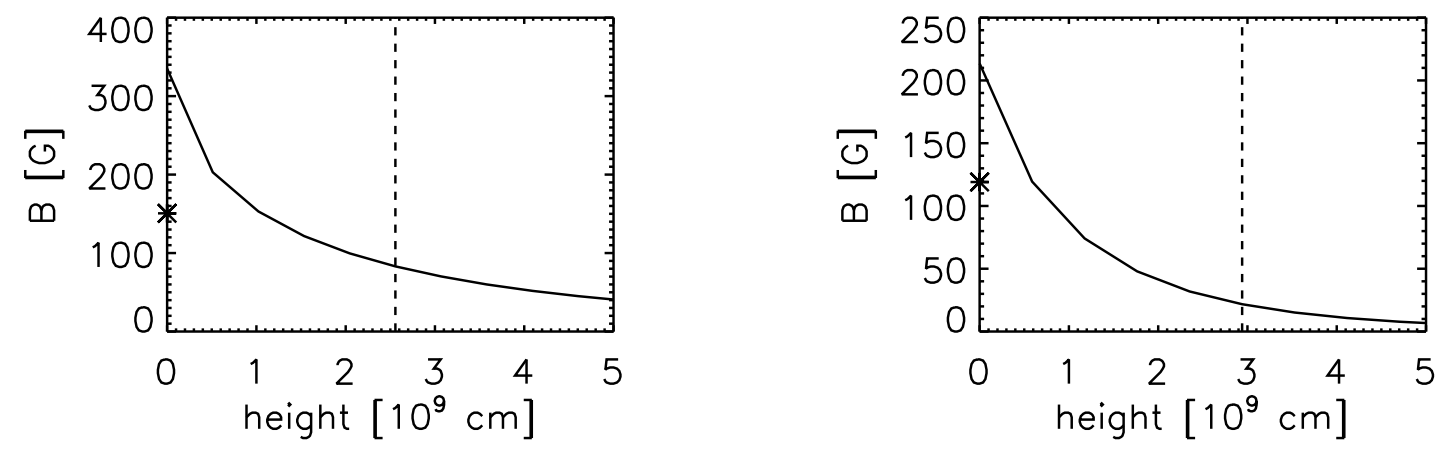

Fig. 7.- Spatial average of potential-field flux density plotted as a function of height. The left panel shows an example of 2000 November 24 X2.3 flare and the other example of 2000 July 14 M3.7 flare is shown in the right panel. The dashed line indicates the size of each flare and the asterisk represents the line-of-sight component of the photospheric flux density.


Fig. 8.- Reconnection rate $M_{\mathrm{A}}$ plotted against the magnetic Reynolds number $R_{m}$. The left panel shows the reconnection rate obtained by method 1, and the right panel shows the reconnection rate obtained by method 2 . In both panels, the dotted lines indicate the dependence of reconnection rate on the magnetic Reynolds number that is predicted by the Petschek model, and the dashed lines indicate the Sweet-Parker-type dependence $M_{\mathrm{A}} \propto R_{m}^{-0.5}$. The dash-dotted line in the left panel indicates the least-squares fitting of the data, which yields $M_{\mathrm{A}} \propto R_{m}^{-0.8}$. Meaning of the symbols is the same as Figure 3 . 